\title{
An Enhanced Efficient Leach Protocol (EELP) with Novel Cross Layer Technique to forward the Data Packets in Wireless Sensor Networks
}

\author{
N.C.Sachithanantham ${ }^{1}$, Dr.V.Jaiganesh ${ }^{2}$ \\ Research Scholar ${ }^{1}$, PG \& Research Department of Computer Science, Dr.NGP College of Arts and Science, \\ Coimbatore - 641048, Tamilnadu, India \\ Professor $^{2}$, PG \& Research Department of Computer Science, Dr.NGP College of Arts and Science, Coimbatore - \\ 641048, Tamilnadu, India \\ sachinphd2020@gmail.com¹, jaiganeshree@gmail.com²
}

\begin{abstract}
Wireless Sensor Networks is one of the leading and broadening research areas by all the researchers since it has got its unique importance in day today's life. This research paper proposed a novel protocol for efficient data forwarding, namely Enhanced Efficient Leach Protocol with Novel Cross-Layer Technique (EELP-NCLT), to forward the data in the WSN. In the proposed structure, the role of $\mathrm{CH}$ is shared by the nodes in the network so the utilization of energy is balanced among the nodes for energy harvesting. A node continues with data transmission to keep up the neutral operation to enhance network lifetime if it does not act as $\mathrm{CH}$. To minimize the network's energy consumption, optimal number of clusters in the network is analyzed. Simulations carried on NS2 Simulator tool exhibit noticeable performance improvements in embracing with proposed EELP-NCLT protocol unlike the conventional scheme in terms of lifetime and network throughput, specifically in constrained conditions. To optimize the performance of relaying, a novel cross-layer TDMA method is additionally exhibited. The network protocol is investigated by considering the performance metrics such as data arrival rate, packet drop ratio, packet delivery ratio, energy consumption, network traffic, delay and network lifetime. The proposed protocol is compared with the baseline techniques. The result shows that the proposed protocol works better than the existing in terms of all performance metrics.
\end{abstract}

Keywords: Clustering, Energy, LEACH, TDMA, PDR, Latency, Network, WSNs.

\section{INTRODUCTION}

The Sensor Network is an infrastructurebased network consisting of elements linked to sensor nodes and battery connectivity. It allows the user the ability to take just-in-time steps to reduce risks. There can be different fields where the WSNs usage is very high such as military, government, hospitals, industries, etc. The sensor network ecosystems are linked to the real-time world linked to the IT architecture. Network-based sensors are identified as a technology that will be significantly enhanced in the next few years with many advanced techniques. WSNs are collections of sensor nodes grouped together in a broad range known as Internet of Things, a dominating area in research. One of the primary difficulties faced in the implementation is its wide deployment in unattended areas and huge energy consumption. Since sensor nodes are dependent on its small battery, many sensors must deploy in a wide area. So to fix this issue, expanding the network's lifetime is a remarkable solution in WSN conventions and data transmissions. Among the other protocols, LEACH is one of the famous hierarchal technique algorithms that partitions network as clusters in which the nodes transmit their packets to $\mathrm{CHs}$ in a Time Division Multiple Access (TDMA) manner. After every TDMA, the cluster head gets aggregated and forwards the packets in a dynamic way from source to sink in the network space. The energy is distributed consistently among every node, which keeps the network balanced every time. But still, the WSN depends upon the successful packet delivery from source to destination; there is a need for a dedicated data forwarding method to make the network more reliable. 
This paper proposes a novel protocol with a duty cycle based clustering method named EELPNCLT that is perfect for sensor networks. In particular, two duty cycles are suggested to keep up an Energy Neutral Operation. Initially, the node embraces a $\mathrm{CH}$ duty cycle for undertaking the role of $\mathrm{CH}$ that is designed by considering the node's capability in harvesting energy. Then for transmitting data, duty cycle that is dependent on nodes energy and data generating range is pursued during non $\mathrm{CH}$ $(\mathrm{NCH})$ rounds. Further, the optimal percentage of $\mathrm{CH}$ (OCHP) is inferred in the network in terms of latency, energy and data transmission requirements. But the OCHP does not always limit the utilization of energy as in the EELP-NCLT protocol. EELP-NCLT has numerous dominance over other techniques that are used in previous systems. To begin with, the selection of $\mathrm{CH}$ in EELP-NCLT is deterministic instead of being founded on a probabilistic methodology that requires various rounds equivalent to a number of nodes before the required level of $\mathrm{CHs}$ is maintained.

In the Wireless Sensor Networks, routing of the data packets from sender to receiver must be well-organized to sustain the protocol's performance and boost the lifetime of the network. Generally, the data packets are transferred from one end to other ends through $\mathrm{CH}$ by forming clusters. Here clustering is achieved by using the optimized clustering algorithm and formulated the problem of optimal $\mathrm{CH}$ range for clustering energy harvesting based networks that ensure Energy Neutral Operation, while fulfilling the requirement of latency and bandwidth. The issue is then resolved by utilizing an iterative strategy for which complexity is limited by the number of nodes in the network.

To keep up the energy harvesting ratio in a better way, $\mathrm{CH}$ selection scheme is proposed that are completely based on duty cycling. This helps select the $\mathrm{CH}$ efficiently even when the energy sources vary frequently in the network space. In LEACH, the number of rounds and nodes must be equal before maintaining the $\mathrm{CH}$ percentage that is required for data transmission. Only the harvesting rates of average nodes are required in the proposed system unlike previous systems. Besides, the proposed convention is appropriate in non-homogeneous systems, in which nodes have distinctive abilities and QoS prerequisites. In proposed, two duty cycles for $\mathrm{CH}$ determination and transmission of data dependent on Energy Neutral Operation are designed.

\subsection{LEACH PROTOCOL}

LEACH protocol alludes to Low Energy Adaptive Clustering Hierarchy [6], which is structured as routing protocol for WSN. Generally, there are three stages in cluster routing, cluster heads generation, cluster formation and communication between clusters. Likewise LEACH incorporates these three stages, simply blending the initial two stages into one that is creation of clusters and cluster communication. Thus, LEACH algorithm contains the cluster setup and stable transmission of data. With respect to the determination of CHs, LEACH follows equal probability strategy, choosing cluster heads randomly and distributes the energy equally among every node in network. Thus LEACH decreases the utilization of energy, maximizes network lifetime. The LEACH procedure is periodical, and every period incorporates the formation of clusters and transmission of data. Here a period is a round is shown in as Figure 1.

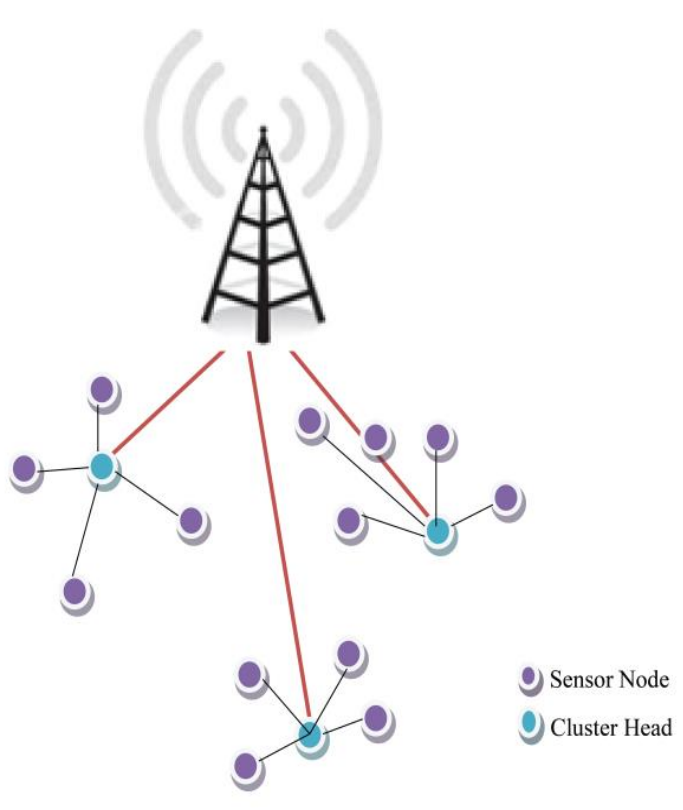

Figure 1: LEACH Protocol 


\subsection{OPTIMIZED CLUSTERING}

Optimized clustering technique depends primarily on Euclidian distances and $\mathrm{CH}$ selection that rely upon residual energies of nodes [7]. Here, the central node gathers the data about node id, position and remaining energy of all nodes and stores this data as a list in the central node. After receiving the required information from all nodes, it begins the process of clustering using the proposed clustering algorithm (k-mean) [8].

\subsubsection{Algorithm}

1. To form the nodes as "k" clusters, initially " $\mathrm{k}$ " number of centroid is taken randomly.

2. Euclidian distance from every node is calculated and assigns it to the nearest centroid.

3. The initial clusters are framed. Assume there are $\mathrm{n}$ nodes such that every single one node belongs to $R_{d}$. The issue of finding minimal variance of these nodes is that of finding $\mathrm{k}$ centroids $\left\{\mathrm{m}_{\mathrm{b}}\right\}^{\mathrm{k}}{ }_{\mathrm{b}=1}$ as follows,

$\left(\frac{1}{n}\right) \times \sum\left(\min d^{2}\left(X_{i}, m_{b}\right)\right)$, for $\mathrm{i}=1$ to $\mathrm{n}$,

where $d\left(X_{i}, m_{b}\right)$ indicates Euclidean distance among $X_{i}$ and $m_{b}\{b\}^{k}{ }_{i=1}$ refers to cluster means or cluster centroids.

4. The centroid position is recalculated in every cluster and the change in centroid position is verified from the previous one.

5. If the change is encountered in centroid position, then step 2 must be followed, else clusters are decided and the process of clustering ends.

\section{LITERATURE SURVEY}

A new way of reconfigurable smart interface sensors introduced for successful delivery of packets in IoT environment [1]. Though it has running in a parallel real-time environment, the life time of network is less due to large capture of data. Classification of energy-efficient protocols study has been examined and compared all the protocols and it is noted that the throughput and packet delivery ratio is less in real time environment [2]. With the availability of limited sensor nodes efficient energy protocols are identified and discussed the challenges of cluster-based protocols [3]. To reduce the energy, the energy-efficient communication protocol is proposed to maximize the network lifetime, but due to its long range, the protocol fails to withstand in the real-time environment [4]. Energy harvesting techniques has been implemented and the author discusses the same to reduce energy [5]. The author proposed a novel LEACH protocol for power efficiency to make the communication more reliable, but due to direct transmissions, the protocol fails to work in large size of networks [6]. The parallel version of the $\mathrm{k}$-means clustering technique has been proposed to provide faster cluster solutions. But it has a disadvantage in enhancing the lifetime of the network [7]. For network operability, the cluster techniques have been proposed and the fuzzy logic system has been imposed to balance the energy consumption, but still the protocol creates an imbalance during the transmission while loading large coverage data [8]. $K$ means cluster algorithm is proposed [9] for enhanced $\mathrm{CH}$ selection via the value of Received Signal Strength (RSS). For a continuous flow of packet delivery alternate choice of $\mathrm{CH}$ is made. Finding the shortest path reduces the consumption of power. Additionally, to reduce the data cost, a compression technique is followed. But the protocol faced an increase in latency time and overhead. This method list routing with the assistance of table from where every node gets most limited path to destination from source is followed [10] but increases traffic and energy consumption. Energy effective routing is divided into four primary plans: Communication Model, Network Structure, Reliable Routing and Topology Based routing [11]. The routing conventions that belong to the second classification can be named Query-based or Coherent and non-lucid based or Negotiation-based. The routing conventions with the third classification can be additionally named Mobile Agent-based or Location-based. The routing conventions having a place with the fourth classification can be additionally divided into Multipath based or QoSbased. An investigative study on energy effective routing conventions for given WSNs is done. In this paper, the characterization is initially proposed and extended to make more energy consumption. New conventions are being used in recent times to perform 
various tasks and effective conveyance in huge traffic. Thus, attention is given to delay and throughput [12]. This article shows WSN MAC conventions' advancement in four classes: synchronous, multi-channel, asynchronous and frame slotted. These structures are assessed by vitality productivity, data conveyance execution, and overhead expected to keep up a convention's systems. In [13] three routing conventions and their issues and difficulties are discussed and compared. RVSRP is proposed to enhance the lifetime of the network and not discussed about the traffic issues in WSNs [14]. EH-WSN [15] is presented for energy harvesting to increase the energy and decrease the latency, but the protocol fails to deliver data successfully on time due to traffic. ECO-LEACH is proposed for duty cycling energy harvesting but fails to dominate the dead node. The broadcast of the network by node increases the error in delivering the data and it maximizes the delay [16-18] The protocols works with large arrange of networks but results indicate that the protocol took more time in capturing the data between nodes and WSN face delay than expected. Multihop energy clustering was proposed to enhance energy consumption, but the protocol fails to work in an isolated area due to continuous overload. The new protocol is proposed to find the alternative route to maximize energy but the overhead is increased.

\section{PROPOSED METHODOLOGY}

Efficient routing must be done for fast communication of data packets in Wireless Sensor network to keep the network's better performance and avoid latency. In our proposed system, the Energy Efficient LEACH protocol is implemented to achieve better routing in the network to achieve the best data transmission. Once the routing is achieved, the data packets are sent as clusters through cluster head $(\mathrm{CH})$. Sink node in network is responsible for sending the data packets to the destination. If the sink node sends the packets directly to the nodes, the chance of data failures is high, which, in turn, increases latency in the network. So the data's are sent to the $\mathrm{CH}$ by the sink node and the $\mathrm{CH}$ directs the packets to a destination via communication nodes. In a network, any node can act as $\mathrm{CH}$, the role of cluster head changes frequently based in the energy level and capacity of node. $\mathrm{CH}$ also monitors each node whether it carries the data packets that are forwarded or if there is any data loss during transmission, this helps to rectify the packet loss, failures and traffic in the network. Additionally, TDMA scheme is been implemented to optimize the network performance and it is bifurcated into slots to check the prospective relays active node transmission and best transmission relays. This helps in analyzing the cluster's bandwidth usage, consumption of energy and latency. Our proposed protocol stands unique from the previous schemes in parameters of data arrival rate, packet drop ratio, packet delivery ratio, energy consumption, network traffic, delay and network lifetime. The three major techniques used such as,

1. Clusters Selection

2. Path Selection

3. Fitness Proportionate Selection

4. EELP-NCLT - Forwarding Data

5. TDMA method

Initially, the node which captured the data will select the nearest node for forwarding the data to reach the clusters, then the end node will select the nearest cluster and sends the captured data. Once the $\mathrm{CHs}$ receive the captured data it will select the finest path for forwarding to reach the destination. It uses nonlinear constrained optimization technique to make the packets to forward. Then the node fitness is calculated in the search space by using the proportionate fitness method. The EELP-NCLT convention task is partitioned into numerous iterations, with each comprising of a short setup stage pursued by a long data transmission stage. In the setup stage of round $\mathrm{t}$, node $\mathrm{z}$ turns into a $\mathrm{CH}$ only if the randomly obtained number is higher than the internal $[0,1]$. The below equation gives it:

$P_{z}(t)= \begin{cases}\frac{\pi}{1-\pi\left(t \bmod \frac{1}{\pi}\right)}, & \text { if } z \in \mathrm{S} \\ 0 & \text { otherwise }\end{cases}$

where $\pi>0$ is the needed percentage of $\mathrm{CHs}$ and $\mathrm{S}$ is the nodes that is not progressed toward becoming $\mathrm{CHs}$ in last $\frac{1}{\pi}$ rounds. This function guarantees that $\mathrm{CH}$ node will never turn into $\mathrm{CH}$ again, until other nodes have fulfilled their turns. Chosen $\mathrm{CHs}$ 
welcome the non- $\mathrm{CH}(\mathrm{NCH})$ nodes to the clusters through beacons. $\mathrm{NCHs}$ will pick the $\mathrm{CHs}$ with the value of best-received signal strength indicator (RSSI) from the invitation beacon and choose their transmit powers $P_{t x}^{C H}$ appropriately. Once the join request is received, $\mathrm{CH}$ broadcasts the schedule of TDMA to its members (CMs), thus finishing the setup stage. The next phase data transmission is comprised of different TDMA outlines wherein for every time slot $T_{s}$, single $C M$ transmits its packets to $\mathrm{CH}$, while rest of $\mathrm{CMs}$ changes to sleep mode. Toward the finish of TDMA frame, $\mathrm{CH}$ sends total packets that are received from CM to sink node at most extreme power $P_{t x}^{\max }$. Every cluster chooses its unique frequency/code channel from available resources to eliminate collision with its neighboring clusters.

In spite of the additions of LEACH, uniform dispersion of $\mathrm{CHs}$ does not give consideration to residual energy of nodes. Thus, the nodes that move toward becoming $\mathrm{CHs}$ first will drain their energies, thus decreasing the system availability that will eventually minimizes network lifetime. In this convention, selection of $\mathrm{CH}$ joins energy harvesting status of every nodes in network to such an extent that the nodes with higher relative rates for harvesting will progress in becoming $\mathrm{CHs}$ more regularly and with no confinement to node in becoming $\mathrm{CH}$. It is expected that every node knows the correct energy status of the considerable number of nodes in the sensor network. Subsequently, the threshold of $\mathrm{CH}$ determination in equation (1) is replaced by:

$P_{Z}(t)=\frac{N \pi\left(E_{Z}^{\text {bat }}+E_{Z}^{\text {har }}\right)}{\sum_{i=1}^{N} E_{i}^{\text {bat }}+E_{i}^{\text {har }}}$

Where $E_{i}^{b a t}$-residual energy

$E_{i}^{\text {har }}$ - harvesting rates of node $\mathrm{i}$

$\mathrm{N}$ - Total nodes in the network.

Where fitness is calculated

$f(x)=N(f) /$ Number of Nodes

CH Selection $=f(x)$ Best fit Node

\subsection{Flow of EELP-NCLT Protocol}

EELP-NCLT protocol carries the following flow process depicted in fig 2. Initially, the formations of network are done, and then formation of cluster and $\mathrm{CH}$ selection is made. Energy utilized by every node is analyzed. If the lifetime of the $\mathrm{CH}$ that is chosen initially ends, the output result is achieved if not the next round starts. Then the process of energy efficiency started in each node by calculating the fitness value. Finally, the TDMA scheme is implemented where the single node or active node sends the data to the $\mathrm{CHs}$ since it is calculated, while other node is in the mute state or sleep state. In the other end $\mathrm{CHs}$ transfer the data to the destination and eliminate the accident or crash between the clusters.

The sink node plays a major role in transferring data, but if the sink node directs the packets directly to selected nodes in the network, there is huge latency and data failure. So clustering technique has been followed by optimized clustering algorithm. The sink node transfers data packets to $\mathrm{CH}$ and then the $\mathrm{CH}$ sends to the destination through nodes. $\mathrm{CH}$ also does the job of monitoring the nodes that carries the data packets to detect the packet drops. The nodes energy capacity and amount of data packets it carries are monitored and reported to sink node. The techniques proposed helps in reducing traffic and latency, increases PDR and avoids packet drops. The optimization of the network is maintained by using TDMA scheme.

\subsection{Novel Cross-Layer Technique}

To maximize the performance of the protocol smart routing is required for effective data transmission between the layers. The cross layer technique allows exchanging the information between the layers with minimal delay. In short it is also called the cross-layer optimization. Here, the $\mathrm{CHs}$ will message the application layer about the energy rating and other surrounding nodes in the WSN. Also other layers will inform about the channel status and resource allocations. During this NCLT the application layer will inform the PHL layer about the next hop information resource. So these types of direct communications will minimize the delay and enhance the timely data transmission in the networks. 
Here the two neighboring layers are $\mathrm{T}_{\mathrm{x}}$ and $\mathrm{T}_{\mathrm{y}}$ and the delay is mentioned as $\mathrm{D}_{T x T y}^{D S}$ and the direct communication is calculated as

$$
\mathrm{D}^{D S} T x T y=\frac{D T x T y}{\mathrm{n}-1}
$$

where $\mathrm{n}-1$ are protocol layers.

\section{IMPLEMENTATION OF NOVEL PROPOSED PROTOCOL}

Implementation is done by analyzing the drawbacks of existing system in forming a network model are rectified and high performance is achieved. Here the major role plays the EELP-NCLT protocol in finding the routing path. To avoid data failure and latency, optimized clustering technique is followed. Our implementation of network model is done NS2 Simulator as it is considered one of the most trusted one in evaluating the performance of protocols. Here the protocols compared are EH-WSN, ECO-LEACH and EELP-NCLT. This research work uses TCL commands and $\mathrm{C}++$ language for NS2 simulation.

\section{PERFORMANCE EVALUATION Table 1: Experimental Simulation Settings}

\begin{tabular}{|l|l|}
\hline \multicolumn{1}{|c|}{ Parameter } & \multicolumn{1}{c|}{ Values } \\
\hline Nodes Count & $500-2000$ \\
\hline Time Slot & $100 \mathrm{~ms}$ \\
\hline Data Packet Rate & 0.5 packets \\
\hline $\begin{array}{l}\text { Initial Transmission Range of } \\
\text { Deployed Nodes }\end{array}$ & $75 \mathrm{~m}$ \\
\hline Type of Traffic & Wireless \\
\hline Initial Energy of Each Node & $20 \mathrm{~J}$ \\
\hline Sensing Range & $10 \mathrm{~m}$ \\
\hline
\end{tabular}

Vol. 4 (7), January 2021, www.ijirase.com

\section{PERFORMANCE METRICS}

Performance metrics used to analyze the proposed EELP-NCLT protocol against EH-WSN and ECOLEACH in terms of data arrival rate, packet drop ratio, packet delivery ratio, energy consumption, network traffic, delay and network lifetime.

\section{RESULT AND DISCUSSIONS}

\subsection{Packet Drop Ratio Analysis}

Packet drop ratio indicates how the packets travel from source to destination in WSNs. Here the packet drop ratio is minimized to $18 \%$ in newly introduced protocol EELP-NCLT when compared to EH-WSN and ECO-LEACH. It is noted that the proposed protocol works better than the existing and delivered the remarkable performance is shown in the Figure 2.

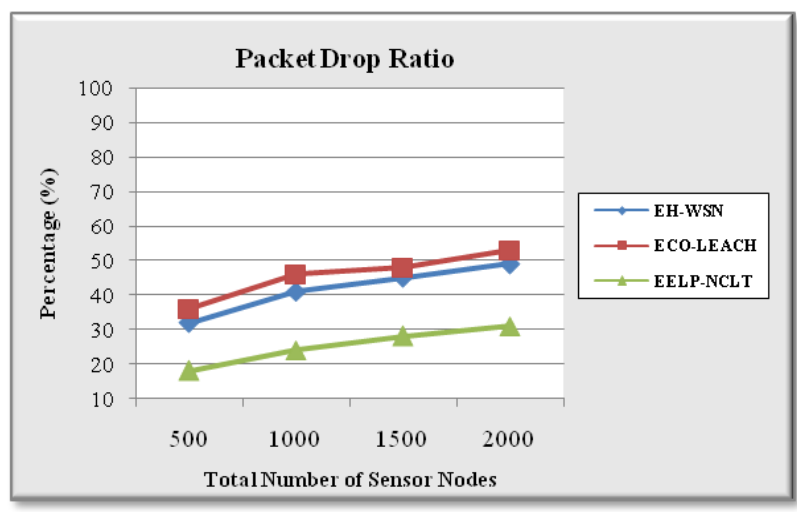

Figure 2: Packet Drop Ratio vs Nodes

\subsection{Packet Delivery Ratio Analysis}

Packet delivery ratio shows that how much percentage of packets or datas has been delivered to the destination without any duplicate or noisy datas. It is noteworthy that EELP-NCLT protocol delivered packets in a successful manner compared to EHWSN and ECO-LEACH protocols. EELP-NCLT protocol shows the delivery rate of $89 \%$ in the result during the simulator execution where other protocols marked only up to $75 \%$ of packet delivery ratio is shown in Figure 3. 


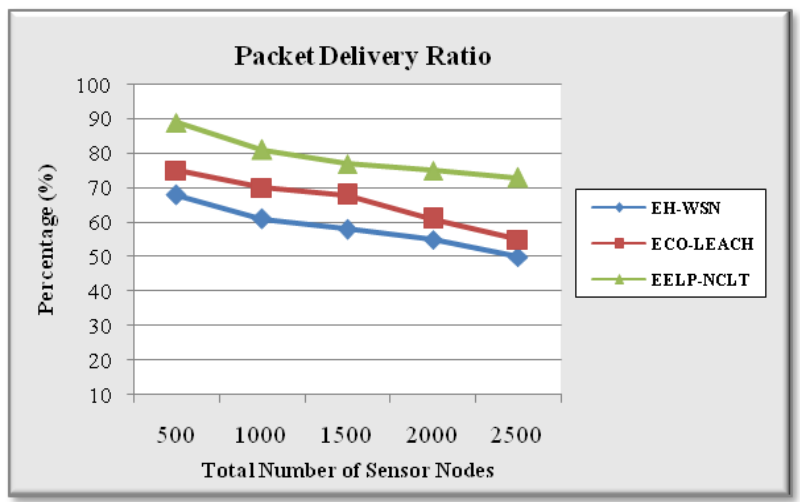

Figure 3: Packet Delivery Ratio vs Nodes

\subsection{Delay Analysis}

Delay is the latency time or time taken for data transmission from one end to another end. Here in this delay analysis it is clearly shown that the proposed protocol EELP-NCLT took minimum time to deliver the packets compared to EH-WSN and ECO-LEACH protocols is shown in Figure 4.

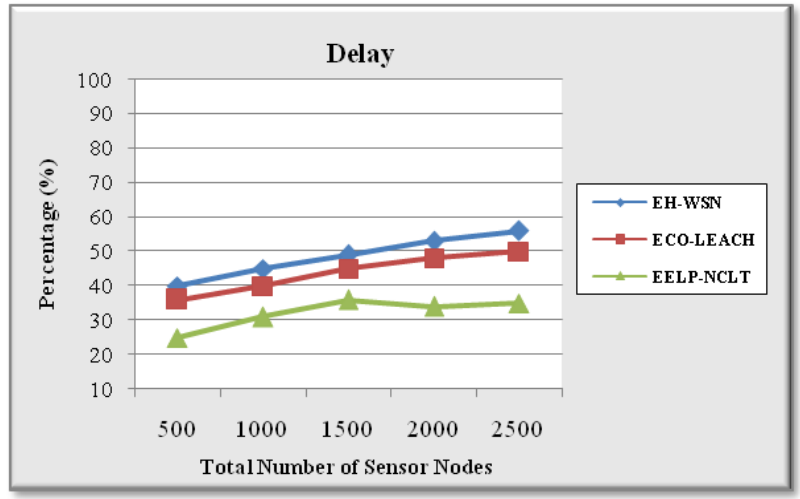

Figure 4: Delay vs Nodes

\subsection{Energy Consumption}

Energy consuption is how much energy is worn out during the data transmission in WSN from one end to other end. In this analysis it is clearly shown that the proposed protocol EELP-NCLT minimize the energy level compared to EH-WSN and ECO-LEACH protocols is shown in Figure 5.

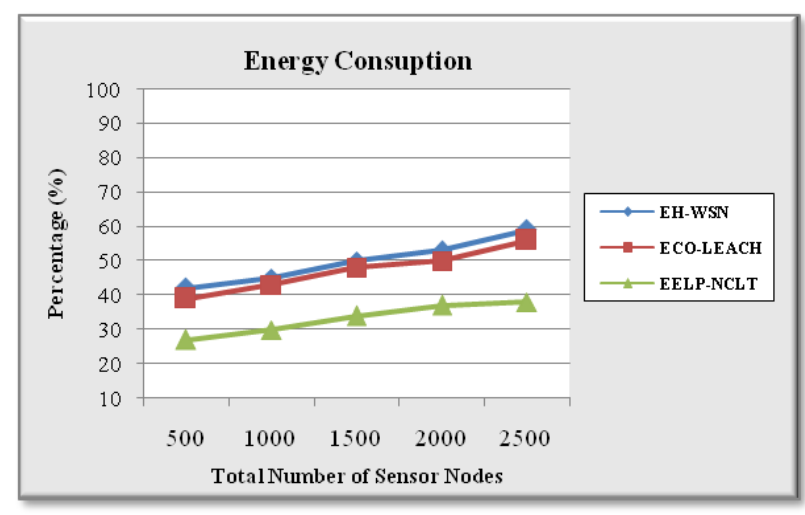

Figure 5: Energy vs Nodes

\subsection{Data Arrival Rate}

Data arrival rate is how much time taken to deliver the data without any duplicates in the woreless sensor networks, It also shows that how much noisy datas captured and eliminated. In this analysis the data delivery rate is high in EELP-NCLT protocol compared to EH-WSN and ECO-LEACH protocols as shown in the Figure 6.

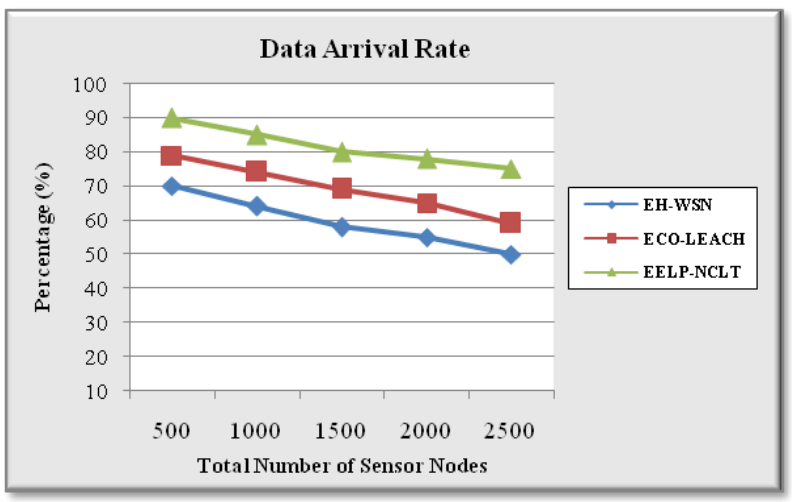

Figure 6: Data Arrival vs Nodes

\subsection{Network Traffic Analysis}

Network Traffic indicates the routing traffic during the data transmission or the amount of data transmitting at a given point of time. In this performance indicator the protocol EELP-NCLT 
shows the high rate in data transmission even the load is high compared to EH-WSN and ECO-LEACH protocols, as shown in Figure 7.

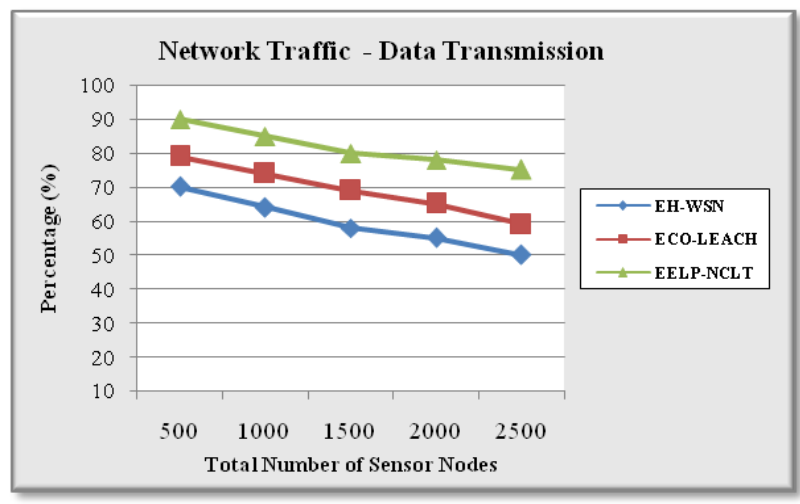

Figure 7: N/W Traffic vs Nodes

\subsection{Network Lifetime Analysis}

Network Lifetime signifies how long the network withstand successfully when the number of nodes increases every time. The network's life span is maximized in this proposed protocol EELP-NCLT up to $92 \%$ compared to EH-WSN and ECO-LEACH protocols as shown in Figure 8.

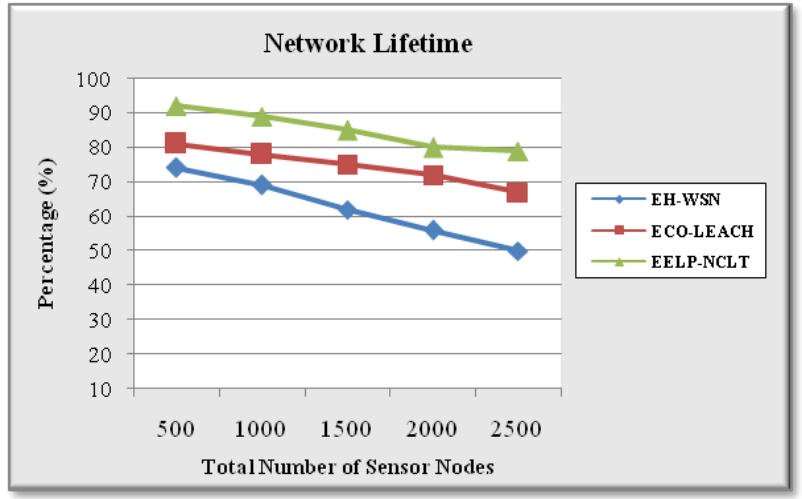

Figure 8: N/W Lifetime vs Nodes

\section{CONCLUSION}

Another energy harvesting method to LEACH convention is proposed called EELP-NCLT dependent on the strategy of duty cycling for assigning $\mathrm{CH}$ ensures with determined percentage and its optimal value was explored. Successful data forwarding is done with optimized routing and the ratio of delay is minimized in this protocol. An effective DC that guarantees consistent operation was developed to transmit data based on nodes harvesting and arrival rates of data. The results acquired utilizing simulations showed improved system execution as far as energy, delay, lifetime and latency regarding benchmark conventions in both EH and battery controlled systems. Future advancements may consider the position of nodes in cycle configuration and also various leveled and multi-hop networks.

\section{REFERENCES}

[1] Chi, Q., Yan, H., Zhang, C., Pang, Z., \& Da Xu, L. (2014). A reconfigurable smart sensor interface for industrial WSN in IoT environment. IEEE transactions on industrial informatics, 10(2), 1417-1425.

[2] Pantazis, N. A., Nikolidakis, S. A., \& Vergados, D. D. (2012). Energy-efficient routing protocols in wireless sensor networks: A survey. IEEE Communications surveys \& tutorials, 15(2), 551591.

[3] Wei, C., Yang, J., Gao, Y., \& Zhang, Z. (2011, December). Cluster-based routing protocols in wireless sensor networks: A survey. In Proceedings of 2011 International Conference on Computer Science and Network Technology (Vol. 3, pp. 1659-1663). IEEE.

[4] Heinzelman, W. R., Chandrakasan, A., \& Balakrishnan, H. (2000, January). Energyefficient communication protocol for wireless microsensor networks. In Proceedings of the 33rd annual Hawaii international conference on system sciences (pp. 10-pp). IEEE.

[5] Benedetti, D., Petrioli, C., \& Spenza, D. (2013, November). GreenCastalia: An energyharvesting-enabled framework for the Castalia simulator. In Proceedings of the 1st International 
Workshop on Energy Neutral Sensing Systems (pp. 1-6).

[6] Lindsey, S., \& Raghavendra, C. S. (2002, March). PEGASIS: Power-efficient gathering in sensor information systems. In Proceedings, IEEE aerospace conference (Vol. 3, pp. 3-3). IEEE.

[7] Joshi, M. N. (2003). Parallel k-means algorithm on distributed memory multiprocessors. Computer, 9.

[8] Forero, P. A., Cano, A., \& Giannakis, G. B. (2011). Distributed clustering using wireless sensor networks. IEEE Journal of Selected Topics in Signal Processing, 5(4), 707-724.

[9] Baranidharan, B., \& Shanthi, B. (2010). A survey on energy efficient protocols for wireless sensor networks. International Journal of Computer Applications, 11(10), 35-40.

[10] Sahoo, B. P. S., Rath, S., \& Puthal, D. (2012). Energy efficient protocols for wireless sensor networks: A survey and approach. International Journal of Computer Applications, 44(18), 43-48.

[11] Pantazis, N. A., Nikolidakis, S. A., \& Vergados, D. D. (2012). Energy-efficient routing protocols in wireless sensor networks: A survey. IEEE Communications surveys \& tutorials, 15(2), 551591.

[12] Huang, P., Xiao, L., Soltani, S., Mutka, M. W., $\&$ Xi, N. (2012). The evolution of MAC protocols in wireless sensor networks: A survey. IEEE communications surveys \& tutorials, 15(1), 101120.

[13] Nithyanandh, S., \& Jaiganesh, V. (2020). Quality of service enabled intelligent water drop algorithm based routing protocol for dynamic link failure detection in wireless sensor network. Indian Jour-nal of Science and Technology, 13(16), 1641-1647.

[14] Tyagi, M. P., \& Jain, M. S. (2012). Comparative study of routing protocols in wireless sensor network. International Journal of Advanced Research in Computer Science and Software Engineering, 2(9).

[15] Nithyanandh, S., \& Jaiganesh, V. (2020). Quality of service enabled intelligent water drop algorithm based routing protocol for dynamic link failure detection in wireless sensor network. Indian Jour-nal of Science and Technology, 13(16), 1641-1647.

[16] Bahbahani, M. S., \& Alsusa, E. (2017). A cooperative clustering protocol with duty cycling for energy harvesting enabled wireless sensor networks. IEEE Transactions on Wireless Communications, 17(1), 101-111.

[17] Peng, S., Wang, T., \& Low, C. P. (2015). Energy neutral clustering for energy harvesting wireless sensors networks. Ad Hoc Networks, 28, $1-16$.

[18] Yang, L., Lu, Y., Zhong, Y., Wu, X., \& Yang, S. X. (2016). A multi-hop energy neutral clustering algorithm for maximizing network information gathering in energy harvesting wireless sensor networks. Sensors, 16(1), 26. 